\title{
Neurobiology of schizophrenia: search for the elusive correlation with symptoms
}

\author{
Daniel H. Mathalon ${ }^{1,2} *$ and Judith M. Ford ${ }^{1,2}$ \\ 1 Psychiatry Service, San Francisco VA Medical Center, San Francisco, CA, USA \\ ${ }^{2}$ Department of Psychiatry, University of California, San Francisco, CA, USA
}

\author{
Edited by: \\ Vince D. Calhoun, University of \\ New Mexico, USA \\ Reviewed by: \\ Kevin M. Spencer, VA Boston \\ Healthcare System/Harvard Medical \\ School, USA \\ Stuart R. Steinhauer, University of \\ Pittsburgh, USA \\ ${ }^{*}$ Correspondence: \\ Daniel H. Mathalon, Psychiatry \\ Service 116D, San Francisco VA \\ Medical Center, 4150 Clement Street, \\ San Francisco, CA 94121, USA. \\ e-mail:daniel.mathalon@ucsf.edu
}

In the last half-century, human neuroscience methods provided a way to study schizophrenia in vivo, and established that it is associated with subtle abnormalities in brain structure and function. However, efforts to understand the neurobiological bases of the clinical symptoms that the diagnosis is based on have been largely unsuccessful. In this paper, we provide an overview of the conceptual and methodological obstacles that undermine efforts to link the severity of specific symptoms to specific neurobiological measures. These obstacles include small samples, questionable reliability and validity of measurements, medication confounds, failure to distinguish state and trait effects, correlation-causation ambiguity, and the absence of compelling animal models of specific symptoms to test mechanistic hypotheses derived from brain-symptom correlations. We conclude with recommendations to promote progress in establishing brain-symptom relationships.

Keywords: schizophrenia, positive symptoms, negative symptoms, neurobiology, correlation, reliability, validity, rating scales
Advances in neuroscience methods over the past 50 years have provided the means to study complex psychiatric disorders in vivo, firmly establishing that disorders once viewed as psychological reactions to stressful environments (particularly family environments) are associated with subtle abnormalities in brain structure and function. This historical transition toward reconceptualizing psychiatric disorders as brain disorders is exemplified by the paradigm shift that gave primacy to neurobiological and neurodevelopmental perspectives in understanding the etiopathology of schizophrenia. Despite the clinical heterogeneity of schizophrenia, a wide variety of neurobiological abnormalities have been replicated across clinical samples and research laboratories, providing some support for the neurobiological validity of the clinical criteria used to diagnose patients. However, efforts to understand the neurobiological bases of the clinical heterogeneity that schizophrenia comprises, mainly by correlating neurobiological measures with specific symptoms, have been largely unsuccessful. Indeed, it is fair to say that "inconsistency" has been the most consistent finding to emerge from such efforts. In this paper, we provide an overview of the myriad conceptual and methodological obstacles that undermine efforts to link the severity of specific symptoms to specific neurobiological measures, obstacles that ultimately impede progress toward elucidating the neurobiological mechanisms underlying these symptoms. We conclude with recommendations to promote progress in establishing brain-symptom relationships.

\section{THE OBSTACLES \\ SMALL SAMPLES}

Neurobiological studies that compare schizophrenia patients to healthy controls often include an analysis of symptom correlations within the patient group. The sample sizes needed to detect mean differences between patients and controls are typically smaller than the sample sizes needed to adequately power symptom correlation studies. The common practice of exploring symptom correlations within the relatively small patient samples employed in typical case-control studies often results in failures to detect significant associations between neurobiological measures and severity ratings of specific symptoms. However, in addition to insufficient power, correlations based on small sample sizes are also susceptible to spurious associations due to the influence that just a few data points can have on the small sample correlation coefficient. While the magnitude of correlations in small samples can be surprisingly large, leading some investigators to assume that the correlation is unlikely to have arisen by chance alone, $95 \%$ confidence intervals estimated for small sample correlations are very wide, reflecting the high level of uncertainty about where the true correlation actually lies (Figure 1).

The problem of small samples is exacerbated by the need to control the type-I error rate for the number of correlations tested, leading to very stringent significance thresholds. However, it is not uncommon for studies that report symptom correlations to forego correction for multiple tests by describing the correlational analyses as "exploratory." The result is that the schizophrenia research literature is replete with inconsistent findings of "significant" correlations with various symptom dimensions based on small patients samples. It is unclear whether these small sample correlations actually move the field forward, or whether they primarily clutter the literature with spurious findings.

What constitutes a small sample? Certainly sample sizes of 30 or less seem small given the need to represent the full range of symptom variation in the patient sample. Sample sizes of 


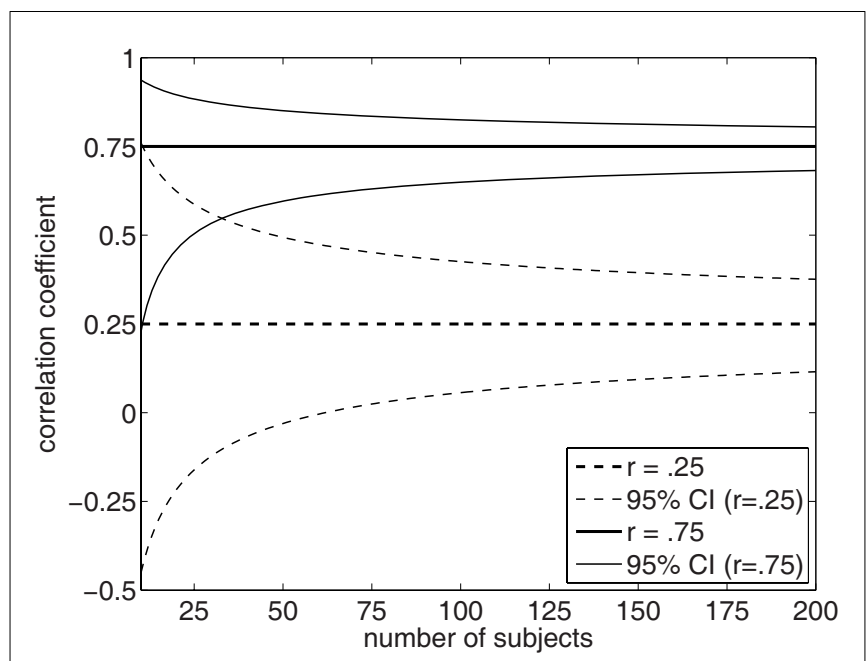

FIGURE 1 |This figure shows the $95 \%$ confidence intervals for two Pearson product-moment correlation coefficient values, $r=0.25$ and $\boldsymbol{r}=\mathbf{0 . 7 5}$, as a function of sample size. Note the wide confidence intervals when correlations are based on small sample sizes, particularly when $n<50$ subjects.

at least 50 patients seem better suited for robust detection of symptom correlations, but such sample sizes are uncommon for neurobiological studies of schizophrenia from individual laboratories.

\section{QUESTIONABLE RELIABILITY AND VALIDITY OF MEASUREMENTS}

Implicit in the assessment of correlations between clinical symptom measures and neurobiological measures is the assumption that both measures possess sufficient reliability and validity to support a meaningful examination of their inter-relationship. However, this assumption is seldom verified empirically. On the clinical side, symptoms are typically quantified using interviewbased rating scales such as the Positive and Negative Symptom Scale (PANSS; Kay et al., 1987), Scale for the Assessment of Positive Symptoms (SAPS; Andreasen, 1984), Scale for the Assessment of Negative Symptoms (SANS; Andreasen, 1983), or Brief Psychiatric Rating Scale (BPRS; Overall and Gorham, 1962, 1988). These scales assess the severity of a specific symptom domain with a single item or a few items, failing to adhere to the psychometric principle that averaging over many items enhances the reliability of psychological construct measurement (Wiggins, 1980; Anastasi, 1982). This sharply contrasts with the typical multi-item approach to measurement taken by psychologists when developing instruments to assess cognitive abilities, personality traits, or other complex psychological constructs.

The problem of unreliability of clinical symptom measurements is exacerbated by the fact that symptoms are typically assessed by interviewers making severity ratings based on semistructured patient interviews. While this calls for considerable skill and judgment on the part of the interviewer, inter-rater reliability is seldom assessed and reported in papers that examine symptom correlations with neurobiological measures. Moreover, although averaging the judgments of multiple raters together can enhance the reliability of measurement, relative to the use of ratings from a single rater (Wiggins, 1980; Anastasi, 1982), this approach is rarely used in schizophrenia research. Even when inter-rater reliability is assessed and reported in clinical studies, it is typically assessed by having two or more raters rate the same set of patient interviews, often derived from a videotape library of prior interviews. This approach inflates the true reliability of interview-based ratings by failing to consider the unreliability introduced by variation across interviewers in their skill and technique in eliciting symptom information from patients. Whether the ratings from two independent raters conducting separate patient interviews are reliable is typically not examined in schizophrenia studies, obscuring what may be a major source of measurement error in the assessment of symptom severity.

In addition to the variation introduced by different interviewers conducting clinical ratings, unreliability also arises from inconsistencies in the way patients endorse and describe specific symptoms, which may interact with the specific interviewer but also with the particular mental state of the patient. Again, the degree to which the reliability of symptom ratings is degraded by patient inconsistencies in reporting is seldom quantified in schizophrenia studies, leading to inflated estimates of the true reliability of symptom ratings. The need for reliable symptom measurements when correlating symptom ratings with neurobiological measures is underscored by the well-known principle that reliability sets the upper limit on validity; that is, assuming that measurement errors are random, a clinical symptom measure cannot be expected to correlate more highly with a neurobiological measure than it correlates with itself (Wiggins, 1980; Anastasi, 1982; de Klerk, 2008).

Aside from reliability concerns in connection with interviewbased symptom rating scales, there is good reason to question whether these ratings are valid reflections of the specific symptom severities they are intended to capture. There are a number of threats to validity of clinical ratings that are seldom discussed in the schizophrenia research literature. For example, schizophrenia patients may have difficulty providing accurate information about their symptoms, including their frequency, duration, severity, and impact on their lives. These difficulties may arise from a lack of insight, deficits in self-reflection and self-monitoring, poor memory when trying to recount the frequency and severity of symptoms that have occurred over prior weeks or months, guardedness and paranoia leading to under-reporting of symptoms, and desire to please the interviewer leading to over- or under-reporting of symptoms. While alternatives to interviewbased retrospective self-reports of symptoms have been described in the literature, including experience sampling methods (Csikszentmihalyi and Larson, 1987; Ben-Zeev et al., 2011; Swendsen et al., 2011; Oorschot et al., 2012) where patients report symptoms in real-time in response to a randomly delivered signal (e.g., pager or phone call) or record symptoms in a diary on a daily basis, these approaches are seldom used in neurobiological studies of schizophrenia. Undoubtedly, such measurements are time consuming and challenging to obtain, but they may provide a more valid picture of the severity of specific symptoms over a defined time period than can be obtained from retrospective reports elicited during an interview. 
Of course, the same concerns about measurement reliability and validity apply to the neurobiological measurements that are correlated with symptom measures in schizophrenia studies. Typically there is no attempt to establish that neurobiological measures derived from neuroimaging or electrophysiological recordings are reliable over time. Yet, this is often an implicit assumption when correlating such measures with ratings that retrospectively integrate symptom severity information over the past week, the past month, or the patient's lifetime. Unreliability of neurobiological measures over relatively short time periods can be expected to attenuate the observed correlations between these measures and symptom severity ratings.

\section{MEDICATION AND TREATMENT RESPONSE CONFOUNDS CLINICAL SEVERITY RATINGS}

Medication status is another factor that introduces noise into the assessment of correlations between symptom severity and neurobiological measures. In most neurobiological studies of schizophrenia, patients are taking stable doses of anti-psychotic medication (and other medications such as antidepressants, mood stabilizers, and anxiolytics) at the time of testing. As a result, ratings of symptom severity reflect variation across patients in the responsiveness of symptoms to treatment, rather than just the variation across patients in the primary pathophysiological processes underlying the severity of specific symptoms. Thus, taking auditory hallucinations as an example, it may be that the neurobiological abnormalities underlying auditory hallucinations will correlate with the severity of untreated hallucinations but not with the severity of residual hallucinations present during chronic anti-psychotic treatment.

In addition, to the extent that patients with more severe or treatment-resistant symptoms receive higher doses of medication, dose-related medication effects can confound the correlation between a neurobiological measure and symptom ratings, potentially introducing spurious correlations between them. While the confounding effects of medication and variation in treatment response can be addressed by studying unmedicated patients, it is generally considered unethical to subject patients to a drug washout solely for the purpose of studying them in an unmedicated state in neurobiological research protocols.

\section{STATE VS. TRAIT CONFUSION}

The natural history of schizophrenia involves fluctuations in the severity of symptoms, particularly positive symptoms, over the illness course. While these fluctuations can be influenced by environmental stressors, they may also be due to pathophysiological fluctuations that have yet to be elucidated. In any case, patients also exhibit trait-like individual differences in symptom severity, with some patients having milder forms of the illness and others being more severely affected. Clinical state fluctuations over time are superimposed on these trait-like individual differences, with each patient fluctuating around his or her own mean level of severity for any given symptom.

Some neurobiological measures, particularly those that reflect brain function and potentially the dynamic neural mechanisms underlying specific symptoms (e.g., ERP or fMRI activation measures), are also likely to show trait-like individual differences and state-related fluctuations over time around each patient's own mean for that measure. In cross-sectional studies that attempt to correlate neurobiological measures with symptom ratings, the trait- and state-related contributions to a patient's symptom severity and neurobiological function are confounded, potentially obscuring a true correlation between state-related changes in symptom severity and neurobiological function. Detection of such correlations requires multi-wave longitudinal assessments in order to model the covariation between a neurobiological measure and symptom severity within each subject without the confound of trait-like individual differences. We successfully demonstrated such an approach in a study that showed fluctuations in the P300 component of the auditory ERP to correlate with positive symptom fluctuations within patients over time (Mathalon et al., 2000). This relationship was not evident in a cross-sectional analysis that attempted to correlate the between-subject differences in P300 and positive symptom severity at a single time point.

Other neurobiological measures that reflect more static characteristics of the brain may not be sensitive to clinical state fluctuations but rather may underlie trait-like individual differences between patients in symptom severity. For example, allelic variation in particular genes may be correlated with trait-like individual differences in the propensity to hallucinate or with the mean severity of hallucinations over the illness course. Such correlations may be significantly attenuated in cross-sectional study designs because the symptom ratings are excessively influenced by the patient's current clinical state. Yet the correlation may become evident if symptom ratings are averaged over multiple occasions over the illness course in order to increase their sensitivity to traitlike individual differences. Averaging over multiple measurement occasions increases the temporal reliability of a symptom measure as predicted by the well-known Spearman-Brown prophecy formula from classical test theory. In practice, the benefit of this approach was demonstrated by Epstein and colleagues in connection with the validation of personality trait measures as predictors of specific behaviors (Epstein, 1984, 1997; Epstein and O'Brien, 1985; Epstein et al., 1996). We similarly showed enhanced detection of the correlation between negative symptoms and P300 amplitude by averaging each of the measures over multiple measurement occasions over the illness course (Mathalon et al., 2000). Similarly, we demonstrated enhanced detection of correlations between progressive gray matter decline in schizophrenia and positive symptom severity by averaging the symptom severity over the baseline and follow-up assessments (Mathalon et al., 2001). In general, studies that examine symptom correlations with neurobiological measures in the schizophrenia literature do not make explicit whether the neurobiological measures posited to underlie specific symptoms should show state-related, or trait-related, covariation with symptom severity. The failure to make this distinction leads to sub-optimal study designs for testing hypotheses about brain-symptom relationships.

\section{CORRELATION-CAUSATION LIMITATIONS}

A significant limitation of clinical research in general, and schizophrenia research in particular, is that we are generally limited to studying pre-existing symptoms and brain abnormalities. Our inability to experimentally manipulate brain mechanisms to 
modulate specific symptoms leads us to rely exclusively on correlational data to evaluate mechanistic models of specific symptoms. Since correlations cannot prove causation, even the demonstration of robust and replicable correlations between a neurobiological measure and symptom severity cannot definitively establish that there is a causal relationship.

\section{THIRD VARIABLES}

Related to the correlation-causation limitation of clinical research studies is the potential for correlations between specific neurobiological measures and specific symptoms to be mediated by "third variables." Often these third variables are not measured in the study, obscuring their role in producing the observed brain-symptom correlation. Examples may include exposures to environmental toxins, abnormalities of specific peptides or proteins, or other pathophysiological mechanisms that have yet to be elucidated. Such variables may give rise to both the measured neurobiological abnormality and the clinical symptom being assessed, and the resulting correlation between the two may have little to do with any underlying causal relationship between them. Moreover, to the extent that the severity of apparently distinct symptoms such as hallucinations and persecutory delusions are correlated, the apparent relationship between a neurobiological measure and one symptom (e.g., hallucinations) may be mediated by its relationship with the correlated symptom (e.g., delusions). Indeed, it remains likely that the actual causal pathophysiological mechanisms that give rise to a number of measured neurobiological abnormalities and a wide range of clinical symptoms, as well as observed correlations between them, have yet to be discovered. This problem fundamentally limits the ability of correlations between neurobiological measures and symptom severity to definitively identify the neurobiological causes of specific symptoms in schizophrenia.

\section{MISTAKEN A PRIORI HYPOTHESES}

A minority of clinical symptom correlation studies in schizophrenia are motivated by specific mechanistic hypotheses about how abnormalities of a specific neurobiological measure should be selectively related to specific symptoms. While hypothesis driven analyses are generally considered to have more scientific value than exploratory "fishing expeditions," conceptually reasonable hypotheses may nonetheless be incorrect. There are numerous examples of this in the schizophrenia literature. Working memory deficits, which compromise the ability to hold information online for short periods of time, have been hypothesized to contribute to formal thought disorder (i.e., disorganized thought process) in schizophrenia (Goldman-Rakic, 1994, 1999), yet relationships between them have not generally been found (but see Perlstein et al., 2001). Similarly, abnormalities in semantic network activations, as reflected by the N400 ERP component, might reasonably be predicted to underlie formal thought disorder, yet often it is delusional thinking rather than thought disorder that correlates with N400 (Debruille et al., 2007). The error-related negativity (ERN), an ERP component elicited by commission errors in choice-response tasks, has been linked to reward processing and reward prediction errors (Goldman-Rakic, 1999; Holroyd and Coles, 2002). Based on this, it is reasonable to predict that ERN abnormalities in schizophrenia should be related to negative symptoms, particularly motivational impairments and anhedonia (Morris et al., 2011). However, reduced ERN amplitude has instead generally been associated with increased positive symptom severity (Mathalon et al., 2002) or the paranoid subtype of schizophrenia in several studies (Kopp and Rist, 1999; Mathalon et al., 2002). Thus, sometimes a priori theoretical models may lead investigators to make the wrong prediction about which symptoms will correlate with a neurobiological measure. While this argues in favor of data-driven exploratory analyses of brain-symptom correlations, the down side of such explorations is the inflated type-I error rate associated with statistical testing of large numbers of correlations.

\section{ABSENCE OF COMPELLING ANIMAL MODELS FOR SPECIFIC SYMPTOMS}

A potentially powerful approach to corroborating specific symptom correlations with neurobiological measures from a mechanistic perspective is to experimentally manipulate the neurobiological measure to induce the symptom in an animal model. Unfortunately, despite the fact that some investigators have speculated that psychotic like symptoms can be observed in non-human primates, there are no compelling animal models for the cardinal symptoms of schizophrenia including auditory hallucinations, delusions, formal thought disorder, and negative symptoms. Relative to other medical diseases that have animal models (e.g., diabetes), the schizophrenia field is hampered by the fact that the symptoms are mainly evident via self-report and language-based communication, neither of which are readily studied in non-human primates or rodents. This fundamentally limits our ability to get scientific traction on the problem of elucidating the neurobiological basis of specific symptoms in schizophrenia. It should be noted that animal models have been developed to study neurobiological measures known to be abnormal in patients in schizophrenia, including ERP components such as mismatch negativity (Javitt et al., 1996; Ehrlichman et al., 2009; Amann et al., 2010) and P50 sensory gating (Freedman et al., 1996; Metzger et al., 2007; Amann et al., 2010). However, these approaches do not overcome the challenges of establishing homologies between animal models and specific clinical symptoms.

\section{POTENTIAL SOLUTIONS AND RECOMMENDATIONS LARGER SAMPLES AND BETTER MEASUREMENTS}

Efforts to find robust correlations between specific symptoms and specific neurobiological measures in schizophrenia would likely achieve more success if such studies employed larger sample sizes where confidence intervals are tighter and spurious correlations are less likely. Inspection of the confidence interval plots shown in Figure 1 suggest that the benefits of larger samples for detecting correlations with confidence starts to diminish after somewhere between 50 and 100 subjects (see Figure 1). In addition, the field needs to make advances in the measurement of symptoms, going beyond the retrospective interview-based ratings of one or a few items that typify the approach taken in current studies. Examples of such approaches include multi-item ratings scales for hallucinations like the psychotic symptom rating scale (PSYRATS; Drake et al., 2007), real-time event sampling to reduce the reliance on patient's retrospective accounts of their symptoms, and assessments of symptoms at multiple time points over the illness course 
to facilitate the distinction between trait- and state-related variation in symptom severity. Finally, greater emphasis needs to be placed on accurate estimation of the reliability of symptom measurements, as well as the reliability of the neurobiological measures that are correlated with symptom severity.

\section{STUDIES OF FIRST-EPISODE MEDICATION-NAIVE PATIENTS AND CHRONIC PATIENTS WHO DO NOT ADHERE TO MEDICATION REGIMENS}

Studies of medication naïve first-episode patients is a potentially useful strategy for capturing variation in primary symptom severity with the confounding effects of medication, but such patients are difficult to recruit and the duration of time that medication can be ethically withheld is fairly limited. Moreover, to the extent that schizophrenia involves a progressive pathophysiology over the illness course, symptom correlations present at the onset of illness may not hold for chronic patients. That symptoms may depend on the stage of illness is supported by studies that suggest that positive symptoms become less severe and negative symptoms more prominent in the later stages of schizophrenia (McGlashan, 1988; McGlashan and Fenton, 1992).

In addition, studying samples of schizophrenia patients who have transiently discontinued their medication or who choose not to take medication is another approach to capturing symptom variation in the absence of medication confounds. However, this approach may not yield symptom correlations that generalize to the entire population of schizophrenia patients, particularly to the extent that chronic patients who can live independently in the community without medication may not be representative of schizophrenia patients in general.

\section{OVERCOMING THE CORRELATION-CAUSATION LIMITATION OF CLINICAL RESEARCH STUDIES}

One strategy that attempts to overcome the limitations of correlational brain-symptom data in clinical research studies is to use pharmacological challenges with agents that affect specific neuroreceptors in order to transiently exacerbate symptoms in patients, or to transiently induce schizophrenia-like symptoms in healthy volunteers. This strategy is exemplified by the use of the NMDA-receptor antagonist, ketamine, in challenge studies with patients (Lahti et al., 1995) and healthy volunteers (Krystal et al., 1994, 2003; Adler et al., 1999; Umbricht et al., 2000; Moore et al., 2011; Neill et al., 2011), to test the hypothesis that NMDAreceptor hypofunction contributes to both clinical symptoms and neurocognitive deficits in schizophrenia.

\section{REFERENCES}

Adler, C. M., Malhotra, A. K., Elman,

I., Goldberg, T., Egan, M., Pickar,

D., and Breier, A. (1999). Compar-

ison of ketamine-induced thought

disorder in healthy volunteers and thought disorder in schizophrenia. Am. J. Psychiatry 156, 1646-1649.

Amann, L. C., Gandal, M. J., Halene, T. B., Ehrlichman, R. S., White, S. L., McCarren, H. S., and Siegel, S. J. (2010). Mouse behavioral endophenotypes for schizophrenia. Brain Res. Bull. 83, 147-161.
Anastasi, A. (1982). Psychological Testing. New York: MacMillan.

Andreasen, N. C. (1983). The Scale for the Assessment of Negative Symptoms (SANS). Iowa City, IA: University of Iowa.

Andreasen, N. C. (1984). Scale for the Assessment of Positive Symptoms. Iowa City, IA: University of Iowa.

Ben-Zeev, D., Ellington, K., Swendsen, J., and Granholm, E. (2011). Examining a cognitive model of persecutory ideation in the daily life of people with schizophrenia: a computerized

Another promising approach to overcoming the correlationcausation conundrum that is still in its early stages of development is the use of transcranial magnetic stimulation (TMS) to transiently perturb specific brain regions and circuits (Slotema et al., 2010). While repetitive TMS has already been studied as a potential treatment for auditory hallucinations (Hoffman et al., 2000), the possibility of using TMS targeted at specific brain regions to transiently increase or decrease specific symptoms in order to provide evidence for the causal role of that brain region in producing or modulating the symptom remains largely unexplored. Nonetheless, both the pharmacological probe and the TMS probe approach to establishing causal connections between brain function and specific symptoms have some conceptual limitations. In particular, even if manipulating brain function with these methods can reproduce schizophrenia-like symptoms, this does not preclude the possibility that distinctly different pathophysiological mechanisms give rise to these symptoms in schizophrenia.

\section{EXTENDING SYMPTOM DIMENSIONS ACROSS TRADITIONAL DIAGNOSTIC BOUNDARIES}

To the extent that some symptoms of schizophrenia are also evident in other neuropsychiatric disorders, studying these symptoms across disorders can provide some leverage against confounds that tend to be more specific to the schizophrenia spectrum. For example, auditory hallucinations can occur in bipolar mania or depression when psychosis accompanies these mood states. Bipolar patients may not have the chronic exposure to anti-psychotic medication typical of schizophrenia, instead being treated with drugs like lithium, valproic acid, and antidepressants. This provides some opportunities to examine symptom correlations with neurobiological measures in bipolar disorder without the confound of dopamine D2 blockade associated with anti-psychotic drugs. Moreover, bipolar patients do not tend to exhibit the negative symptoms or severe functional impairment characteristic of schizophrenia, allowing further dissociation of what tend to be correlated impairments in schizophrenia. Indeed, linking symptoms to brain circuits across traditional diagnostic boundaries has been identified as a major research initiative by $\mathrm{NIMH}$, the so-called Research Domain Criteria (RDoC) initiative. This approach may provide a greater range of variability for specific symptoms and neurobiological abnormalities that can enhance the likelihood of finding significant covariation between them.

experience sampling study. Schizophr. Bull. 37, 1248-1256.

Csikszentmihalyi, M., and Larson, R. (1987). Validity and reliability of the experience-sampling method. J. Nerv. Ment. Dis. 175, 526-536.

de Klerk, G. (2008). "Classical test theory (CTT)," in Online Readings in Testing and Assessment, International Test Commission, eds M. Born, C. D. Foxcroft, and R. Butter. Availiable at: http://www.intestcom.org/ Publications/ORTA.php
Debruille, J. B., Kumar, N., Saheb, D., Chintoh, A., Gharghi, D., Lionnet, C., and King, S. (2007). Delusions and processing of discrepant information: an event-related brain potential study. Schizophr. Res. 89, 261-277.

Drake, R., Haddock, G., Tarrier, N., Bentall, R., and Lewis, S. (2007). The psychotic symptom rating scales (PSYRATS): their usefulness and properties in first episode psychosis. Schizophr. Res. 89, 119-122. 
Ehrlichman, R. S., Luminais, S. N., White, S. L., Rudnick, N. D., Ma, N., Dow, H. C., Kreibich, A. S., Abel, T., Brodkin, E. S., Hahn, C. G., and Siegel, S. J. (2009). Neuregulin 1 transgenic mice display reduced mismatch negativity, contextual fear conditioning and social interactions. Brain Res. 1294, 116-127.

Epstein, S. (1984). A procedural note on the measurement of broad dispositions. J. Pers. 52, 318-325.

Epstein, S. (1997). This I have learned from over 40 years of personality research. J. Pers. 65, 3-32.

Epstein, S., and O’Brien, E. J. (1985). The person-situation debate in historical and current perspective. Psychol. Bull. 98, 513-537.

Epstein, S., Pacini, R., Denes-Raj, V., and Heier, H. (1996). Individual differences in intuitive-experiential and analytical-rational thinking styles. J. Pers. Soc. Psychol. 71, 390-405.

Freedman, R., Adler, L. E., MylesWorsley, M., Nagamoto, H. T., Miller, C., Kisley, M., McRae, K., Cawthra, E., and Waldo, M. (1996). Inhibitory gating of an evoked response to repeated auditory stimuli in schizophrenic and normal subjects. Human recordings, computer simulation, and an animal model. Arch. Gen. Psychiatry 53, 1114-1121.

Goldman-Rakic, P. (1994). Working memory dysfunction in schizophrenia. J. Neuropsychiatry Clin. Neurosci. 6, 348-357.

Goldman-Rakic, P. (1999). The physiological approach: functional architecture of working memory and disordered cognition in schizophrenia. Biol. Psychiatry 46, 650-661.

Hoffman, R., Boutros, N., Hu, S., Berman, R., Krystal, J., and Charney, D. (2000). Transcranial magnetic stimulation and auditory hallucinations in schizophrenia. Lancet 355, 1073-1075.

Holroyd, C., and Coles, M. G. H. (2002). The neural basis of human error processing: reinforcement learning, dopamine and the errorrelated negativity. Psychol. Rev. 109, 679-709.

Javitt, D. C., Steinschneider, M., Schroeder, C. E., and Arezzo, J. C.
(1996). Role of cortical N-methyl$\mathrm{D}$-aspartate receptors in auditory sensory memory and mismatch negativity generation: implications for schizophrenia. Proc. Natl. Acad. Sci. U.S.A. 93, 11962-11967.

Kay, S., Fiszbein, A., and Opler, L. (1987). The positive and negative syndrome scale (PANSS) for schizophrenia. Schizophr. Bull. 13, 261-276.

Kopp, B., and Rist, F. (1999). An eventrelated brain potential substrate of disturbed response monitoring in paranoid schizophrenic patients. $J$. Abnorm. Psychol. 108, 337-346.

Krystal, J. H., D’Souza, D. C., Mathalon, D., Perry, E., Belger, A., and Hoffman, R. (2003). NMDA receptor antagonist effects, cortical glutamatergic function, and schizophrenia: toward a paradigm shift in medication development. Psychopharmacology (Berl.) 169, 215-233.

Krystal, J. H., Karper, L. P., Seibyl, J. P., Freeman, G. K., Delaney, R., Bremner, J. D., Heninger, G. R., Bowers, M. B. Jr., and Charney, D. S. (1994). Subanesthetic effects of the noncompetitive NMDA antagonist, ketamine, in humans. Psychotomimetic, perceptual, cognitive, and neuroendocrine responses. Arch. Gen. Psychiatry 51, 199-214.

Lahti, A. C., Koffel, B., LaPorte, D., and Tamminga, C. A. (1995). Subanesthetic doses of ketamine stimulate psychosis in schizophrenia. Neuropsychopharmacology 13, 9-19.

Mathalon, D. H., Fedor, M., Faustman, W. O., Gray, M., Askari, N., and Ford, J. M. (2002). Response-monitoring dysfunction in schizophrenia: an event-related brain potential study. J. Abnorm. Psychol. 111, 22-41.

Mathalon, D. H., Ford, J. M., and Pfefferbaum, A. (2000). Trait and state aspects of $\mathrm{P} 300$ amplitude reduction in schizophrenia: a retrospective longitudinal study. Biol. Psychiatry 47, 434-449.

Mathalon, D. H., Sullivan, E. V., Lim, K. O., and Pfefferbaum, A. (2001). Progressive brain volume changes and the clinical course of schizophrenia: a longitudinal magnetic resonance imaging study. Arch. Gen. Psychiatry 58, 148-157.

McGlashan, T. H. (1988). A selective review of recent North American long-term followup studies of schizophrenia. Schizophr. Bull. 14, 515-542.

McGlashan, T. H., and Fenton, W. S. (1992). The positive-negative distinction in schizophrenia - review of natural history validators. Arch. Gen. Psychiatry 49, 63-72.

Metzger, K. L., Maxwell, C. R., Liang, Y., and Siegel, S. J. (2007). Effects of nicotine vary across two auditory evoked potentials in the mouse. Biol. Psychiatry 61, 23-30.

Moore, J. W., Turner, D. C., Corlett, P. R., Arana, F. S., Morgan, H. L., Absalom, A. R., Adapa, R., de Wit, S., Everitt, J. C., Gardner, J. M., Pigott, J. S., Haggard, P., and Fletcher, P. C. (2011). Ketamine administration in healthy volunteers reproduces aberrant agency experiences associated with schizophrenia. Cogn Neuropsychiatry 1-18.

Morris, S. E., Holroyd, C. B., MannWrobel, M. C., and Gold, J. M. (2011). Dissociation of response and feedback negativity in schizophrenia: electrophysiological and computational evidence for a deficit in the representation of value. Front. Hum. Neurosci. 5:123. doi:10.3389/fnhum.2011.00123

Neill, E., Rossell, S. L., McDonald, S. Joshua, N., Jansen, N., and Morgan, C. J. (2011). Using ketamine to model semantic deficits in schizophrenia. J. Clin. Psychopharmacol. 31, 690-697.

Oorschot, M., Lataster, T., Thewissen, V. Wichers, M., and Myin-Germeys, I. (2012). Mobile assessment in schizophrenia: a data-driven momentary approach. Schizophr. Bull. 38, 405-413.

Overall, J. E., and Gorham, D. R. (1962). The brief psychiatric rating scale. Psychol. Rep. 10, 799-812.

Overall, J. E., and Gorham, D. R (1988). The brief psychiatric rating scale (BPRS): recent developments in ascertainment and scaling. Psychopharmacol. Bull. 24, 97-99.
Perlstein, W. M., Carter, C. S., Noll, D. C., and Cohen, J. D. (2001). Relation of prefrontal cortex dysfunction to working memory and symptoms in schizophrenia. A. J. Psychiatry 158, 1105-1113.

Slotema, C. W., Blom, J. D., Hoek, H. W., and Sommer, I. E. (2010). Should we expand the toolbox of psychiatric treatment methods to include repetitive transcranial magnetic stimulation (rTMS)? A meta-analysis of the efficacy of rTMS in psychiatric disorders. J. Clin. Psychiatry 71, 873-884.

Swendsen, J., Ben-Zeev, D., and Granholm, E. (2011). Real-time electronic ambulatory monitoring of substance use and symptom expression in schizophrenia. Am. J. Psychiatry 168, 202-209.

Umbricht, D., Schmid, L., Koller, R., Vollenweider, F. X., Hell, D., and Javitt, D. C. (2000). Ketamineinduced deficits in auditory and visual context-dependent processing in healthy volunteers: implications for models of cognitive deficits in schizophrenia. Arch. Gen. Psychiatry 57, 1139-1147.

Wiggins, J. S. (1980). Personality and Prediction: Principles of Personality Assessment. Reading, MA: AddisonWesley Publishing Company.

Conflict of Interest Statement: The authors declare that the research was conducted in the absence of any commercial or financial relationships that could be construed as a potential conflict of interest.

Received: 15 January 2012; accepted: 29 April 2012; published online: 25 May 2012.

Citation: Mathalon DH and Ford JM (2012) Neurobiology of schizophrenia: search for the elusive correlation with symptoms. Front. Hum. Neurosci. 6:136. doi: 10.3389/fnhum.2012.00136

Copyright (c) 2012 Mathalon and Ford. This is an open-access article distributed under the terms of the Creative Commons Attribution Non Commercial License, which permits non-commercial use, distribution, and reproduction in other forums, provided the original authors and source are credited. 